\title{
Social Determinants of Mental Health and Role of Social Inequalities
}

\author{
A,Mohseni-Bandpei ${ }^{1, *}$ \\ ${ }^{1}$ Professor in Environmental Health, School of Public Health, Shahid Beheshti University, President of State Welfare Organization \\ ${ }^{*}$ Corresponding author: Anoushiravan Mohseni-Bandpei, Professor in Environmental Health, School of Public Health, Shahid Beheshti University, President of State Welfare Orga- \\ nization, Tehran, Iran. Email: A.mohseni8@yahoo.com
}

Received: 22 Sep 2016

Accepted: 21 Nov 2016

Ppub: 23 Dec 2016

\begin{abstract}
Background: Mental health is one the most important parts of health and wellbeing of human beings. Since too many years ago and now more than ever, respect to mental health are emphasized. Community mental health and many mental disorders are under effects of different social, economical and physical environments. Social inequalities can lead to risk factors in mental health and mental disorders. More social inequalities, more risk factors inequalities, and then more mental disorders.

Methods: According to country studies, prevalence of mental disorders in populations between 15 and 60 years is $23.6 \%$. Also from international studies almost $50 \%$ and $75 \%$ of mental disorders begin before 14 years old and 24 years old respectively.

Results: So it is important that interventions for improvement of social conditions, and reducing social inequalities begin before birth and continue to early child years, at school age, during family building and working ages, and at older ages.

Conclusions: Interventions in social determinants across the life course, not only are valuable opportunities for community mental health promotion, but can lead to reduction of mental health and mental disorders risk factors due to social inequalities.

Keywords: Social Inequalities; Mental Health; Mental Disorders; Social Determinants
\end{abstract}

